\title{
Adult mood problems in children with neurodevelopmental problems: evidence from a prospective birth cohort followed to age 50
}

\author{
Alishia Addicoat ${ }^{1} \cdot$ Ajay K. Thapar ${ }^{1} \cdot$ Lucy Riglin $^{1} \cdot$ Anita Thapar $^{1} \cdot$ Stephan Collishaw $^{1}$ (1)
}

Received: 15 November 2018 / Accepted: 13 May 2019 / Published online: 22 May 2019

(c) The Author(s) 2019

\begin{abstract}
Purpose Specific child neurodevelopmental (ND) disorders such as ADHD and learning problems are associated with concurrent and future (up to early adulthood) mood problems. However, it is unclear whether findings generalise to population traits as well as diagnoses, to general as well as specific neurodevelopmental domains, and whether risk associations extend to later adulthood or diminish with age.

Methods We used data from a UK cohort of children born in 1958, the National Child Development Study (NCDS). ND problems were assessed at ages 7 and 11 years with parent- and teacher ratings of restlessness, hyperactivity and motor coordination difficulties, and by individual tests of reading, arithmetic and general cognitive ability. Mood (depression/anxiety) problems were assessed using the Malaise symptom screen at 23, 33, 42, and 50 years. Factor analyses were conducted to assess whether the specific neurodevelopmental domains could be aggregated into a general "ND" latent factor as well as specific factors. Associations with mood outcomes were then tested.

Results A bi-factor model with a general "ND" latent factor and specific "motor" and "cognition" factors fits the data well. The specific cognition and motor factor scores were associated with mood problems in early adulthood only. The "ND" factor demonstrated associations with mood problems at each adult follow-up (men - age 23 years: $\beta=0.17$; age 33: $\beta=0.16$; age 42 : $\beta=0.14$; age 50 : $\beta=0.16$; women -23 years: $\beta=0.25$; 33 years: $\beta=0.26$; 42 years: $\beta=0.14 ; 50$ years: $\beta=0.16$; all $p \mathrm{~s}<0.01)$. Interactions by sex indicated that the association between this general factor and mood problems was more pronounced for women than men at ages 23 years $(\beta=0.09, p=0.005)$ and 33 years $(\beta=0.10, p=0.003)$, but not at 42 or 50 years $(p s>0.8)$.

Conclusions Our results suggest that, in a population-based cohort, a general, childhood neurodevelopmental difficulty factor is stably associated with mood problems in adult life.
\end{abstract}

Keywords Neurodevelopmental $\cdot$ Childhood $\cdot$ Longitudinal $\cdot$ Adult depression $\cdot$ Bi-factor

\section{Introduction}

Childhood neurodevelopmental disorders include a broad range of conditions that involve neurocognitive, motor, and social deficits [1]. These include attention-deficit/

Electronic supplementary material The online version of this article (https://doi.org/10.1007/s00127-019-01727-5) contains supplementary material, which is available to authorized users.

Stephan Collishaw

collishaws@cardiff.ac.uk

1 Division of Psychological Medicine and Clinical Neurosciences, MRC Centre for Neuropsychiatric Genetics and Genomics, Cardiff University School of Medicine, Child and Adolescent Psychiatry, Hadyn Ellis Building, Maindy Road, Cardiff CF24 4HQ, Wales, UK hyperactivity disorder, developmental co-ordination disorder, autism spectrum disorder, intellectual disability, and specific learning disorders. These are viewed as categories for clinical purposes, but have been found to behave as traits in epidemiological and genetic studies [2]. Neurodevelopmental disorders and traits show a high degree of co-occurrence/correlation, are more prevalent in males than females, and demonstrate a similar developmental course involving pre-pubertal onset and a stable rather than episodic presentation [2]. One possible explanation for these common characteristics is that neurodevelopmental problems share a common underpinning general liability.

Besides the core symptoms, children with neurodevelopmental problems tend to experience a wide range of other psychiatric and functional problems in childhood, adolescence, and the early adult years [3-5]. This includes an 
elevated rate of mood problems [6-8]. Adult mood problems such as depression and anxiety collectively constitute the world-leading causes of years-lived-with-disability [9]. Early prevention of depression is important but interventions typically focus on well-established childhood risk factors that include negative life events and family history of depression [10] rather than neurodevelopmental problems.

Most previous longitudinal studies have concentrated on specific neurodevelopmental disorders in isolation (e.g. ADHD, developmental co-ordination disorder, or intellectual disability) and in some cases excluded individuals with other co-occurring neurodevelopmental problems $[6,7]$. Thus, these studies do not reflect what is typically observed-strong co-occurrence between different neurodevelopmental problems. There is growing evidence that high levels of co-occurrence between different indices of psychopathology may be underpinned by a general psychopathology factor (' $p$ ', a term coined by Caspi et al. [11]) and behavioural, emotional as well as neurodevelopmental traits have been found to load on this general factor [12, 13]. This approach has also been used to hone in on patterns of co-occurrence of neurodevelopmental traits where evidence shows loading of specific traits onto one general neurodevelopmental factor $[14,15]$, as well as several sub-factors broadly indexing motor, learning and autism-related phenotypes [15]. We are not aware of any studies that have tested general or trait-specific links between neurodevelopmental problems and later adult mood problems.

A second limitation is that the majority of previous research has measured outcomes in early adulthood, whereas mid-life and onwards has been neglected. Understanding whether associations with mood problems persist later into adulthood or diminish with time is important for informing both theory and practice. It is possible that a general neurodevelopmental liability may index increased life-long risk for mood problems despite specific symptom presentations such as hyperactivity or co-ordination problems that often improve with maturity [16]. Such specific symptom presentations might only show relatively time-limited associations with adult mood.

A final problem is that much of the previous literature is based on clinic-based samples. These can introduce referral biases and may not include sub-threshold cases. We address these issues by capitalising on a large UK population-based cohort (the National Child Development Study) with multiinformant trait-based measures of neurodevelopmental problems in childhood and repeated data on self-reported mood problems in adulthood. The study includes multiple measures of two core neurodevelopmental domains-a motor domain (restlessness, hyperactivity, co-ordination problems) and a cognitive domain (reading, maths, general cognitive ability). The study did not include requisite measures of ASD-related symptoms.
The study aims were to (1) test whether childhood neurodevelopmental problems, as assessed in NCDS, load onto a latent general neurodevelopmental factor as well as on specific motor and cognition factors; and (2) investigate associations between general and trait-specific factors with mood problems in early, middle and later adulthood (23-50 years). We hypothesised that long-term associations with mood problems would at least in part be driven by a general neurodevelopmental factor.

\section{Methods}

\section{Sample}

NCDS includes data from a UK cohort comprising children born 3-9 March in 1958 in England, Scotland and Wales [17]. The sample was assessed repeatedly in childhood and adulthood. Here we focus on data collected from the study children and their parents and teachers at ages 7 and 11 years, and from cohort members at ages 23, 33, 42, and 50 years. For those with relevant data during childhood $(N=10054)$, outcome data were available for 9330 at age 23,8380 at age 33,8452 at age 42 and 7253 at age 50. All data sets used in the present study were accessed via the UK data archive, and are covered by the study's original research ethical approvals [18].

\section{Measures}

\section{Childhood neurodevelopmental problems}

The cohort included information from parents, teachers and children about six indicators of neurodevelopmental functioning: (1) restlessness, (2) hyperactivity, (3) developmental co-ordination difficulties; (4) reading attainment, (5) mathematical attainment; (6) general cognitive ability. We were unable to index symptoms of autism spectrum, tic or communication disorders for the purpose of this study. Child restlessness was assessed using the Bristol Social Adjustment Guides (BSAG) completed by teachers at ages 7 and 11 [19]. Four items were rated as absent (0) or present (1) (e.g. 'too restless to work alone'; 'lacks persistence'; [20]). Scores ranged from 0 to 4 and were averaged across age. Child hyperactivity was assessed using the parent and teacher Rutter behaviour questionnaires at ages 7 and 11 years [21]. The Rutter scale included two items ('difficulty settling' and 'squirmy, fidgety behaviour') each rated 'not true' (0), 'somewhat true' (1) or 'certainly true' (2) with summed scores for each informant ranging $0-4$. We computed the average hyperactivity score across both informants and both ages. Childhood motor co-ordination problems were assessed using teacher-completed questionnaires comprising 
four items at ages 7 and 11 years (e.g. 'poor hand control', 'clumsy'; [22]). Each item was rated as 'not true' (0), 'somewhat true' (1) or certainly true' (2). Mean co-ordination problem scores were averaged across age. Child reading and maths attainment were measured at age 7 using the 30 -item Southgate Group Reading Test [23] and the 10-item Problem Arithmetic Test [24]. Child general cognitive ability was assessed at age 11 years using the classroom-based 80-item General Ability Test [25] which included 40 verbal and 40 non-verbal items. Each of the six continuous indicator variables (hyperactivity, restlessness, co-ordination problems, reading attainment, maths attainment, general cognitive ability) was standardised using $z$ scores.

\section{Adult mood problems}

Adult mood was assessed using the self-report Malaise Inventory at ages 23, 33, 42 and 50 years. The Malaise Inventory is an established measure of mood problems in adults, previously validated against interview-assessed major depressive disorder [26]. Individual items (e.g. 'Do you often feel miserable or depressed?') were rated as 'no' (0) or 'yes' (1). As in previous studies using this cohort [27, 28], we used the 15-item psychological distress subscale to index mood problems at ages 23, 33 and 42 (internal consistency $\alpha=0.75-0.87)$ and the nine-item version of the Malaise Inventory available at age $50(\alpha=0.79)$. Malaise scores at each age were standardised using $z$ scores for analysis.

\section{Sensitivity analyses}

Additional analyses examined the influence on associations of adjusting for childhood conduct and emotional problems. These were assessed using three items each from the mothercompleted Rutter A symptom questionnaire at age 7 [21]. Individual items were rated as 'never' (0), 'sometimes' (1), or 'frequently' (2). Age 7 conduct problem (range 0-6) and emotional problem (range 0-6) scale scores were standardised using $z$ scores.

\section{Analysis}

Confirmatory factor analysis using the statistical modelling program, MPlus (version 8) was carried out. Three alternative measurement models were compared: (1) a unidimensional, general factor model with a single latent factor indexed by all six indicator variables (hyperactivity, restlessness, co-ordination problems, reading, maths, cognitive ability); (2) a correlated factors model with two latent factors ('motor' indexed by hyperactivity, restlessness, coordination problems, and 'cognition' indexed by reading, maths, cognitive ability); (3) a bi-factor model (a general ND factor indexed by all six indicators as well as orthogonal motor and cognition factors). Model fit indices were compared across the three alternative model specifications and the best-fitting model chosen. Model fit was considered satisfactory if RMSEA $<0.06, \mathrm{CFI}>0.95$ and TFI $>0.95$ [29]. Sample size-adjusted Bayesian Information Criteria are reported to allow for comparison across alternative models. We then tested associations between the latent factors and mood problems at ages 23, 33, 42 and 50 years. Given high co-occurrence between childhood neurodevelopmental, emotional and conduct problems, secondary analyses evaluated the potential impact of co-occurring childhood conduct and emotional problems on associations between the identified neurodevelopmental factors and adult mood problems. Analyses of adult outcomes incorporated previously derived sweep-specific inverse probability weights shown to minimise measured biases related to non-response at ages 23, 33, 42 and 50 years [27]. Given well-established sex differences in rates of child neurodevelopmental (more prevalent in males) and adult mood problems (more prevalent in females), analyses tested for interactions by sex to test differences in associations between childhood neurodevelopmental problems and adult mood problems for males and females.

\section{Results}

\section{Measurement models}

Correlations for the variables included in the measurement model are shown in the Supplementary material (S1). Factor loadings for the unidimensional, correlated two-factor and bi-factor models are shown in Fig. 1 (panels a-c). The bifactor model (panel c) provided the best fit to the data (Chisq $(24)=35.2 ;$ RMSEA $=0.026(0.018-0.034)$; CFI $=0.998$; $\mathrm{TLI}=0.991$; sample adjusted $\mathrm{BIC}=227024)$. Fit indices were worse for the correlated factors model (panel $b$ ); Chisq $(21)=591.6$; RMSEA $=0.067(0.062-0.071)$; CFI $=0.966$; $\mathrm{TLI}=0.936$; sample adjusted $\mathrm{BIC}=227580$ and the singlefactor model (panel a); Chisq (18) $=1803.6$; RMSEA $=0.111$ (0.106-0.115); CFI $=0.895$; $\mathrm{TLI}=0.825$; sample adjusted $\mathrm{BIC}=229231$ ). Within the bi-factor model, higher general factor scores represented poorer reading, maths and general ability and greater hyperactivity, restlessness and coordination problems.

\section{Associations with adult mood problems}

Using the bi-factor model specification, analyses tested associations between the general and specific (motor and cognition) factors with adult mood problems at ages 23, 33, 42 and 50 years. As summarised in Table 1, the general neurodevelopmental factor showed associations with mood 
Fig. 1 Comparison of singlefactor (a), correlated-factor (b) and bi-factor (c) models with six indicator variables (restlessness, hyperactivity, motor co-ordination problems, reading ability, arithmetic ability, general cognitive ability)
Panel A



Panel B

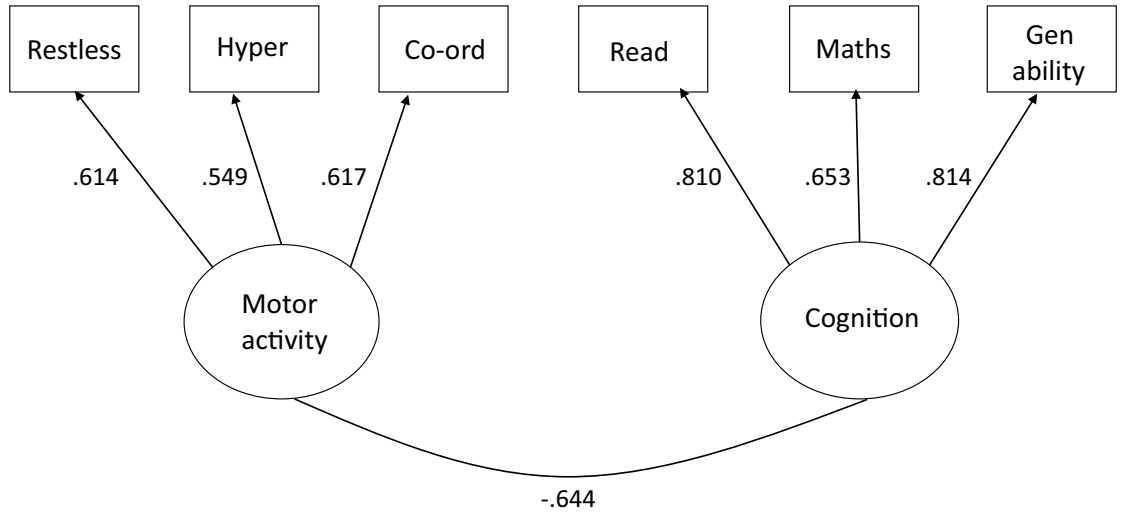

Panel C

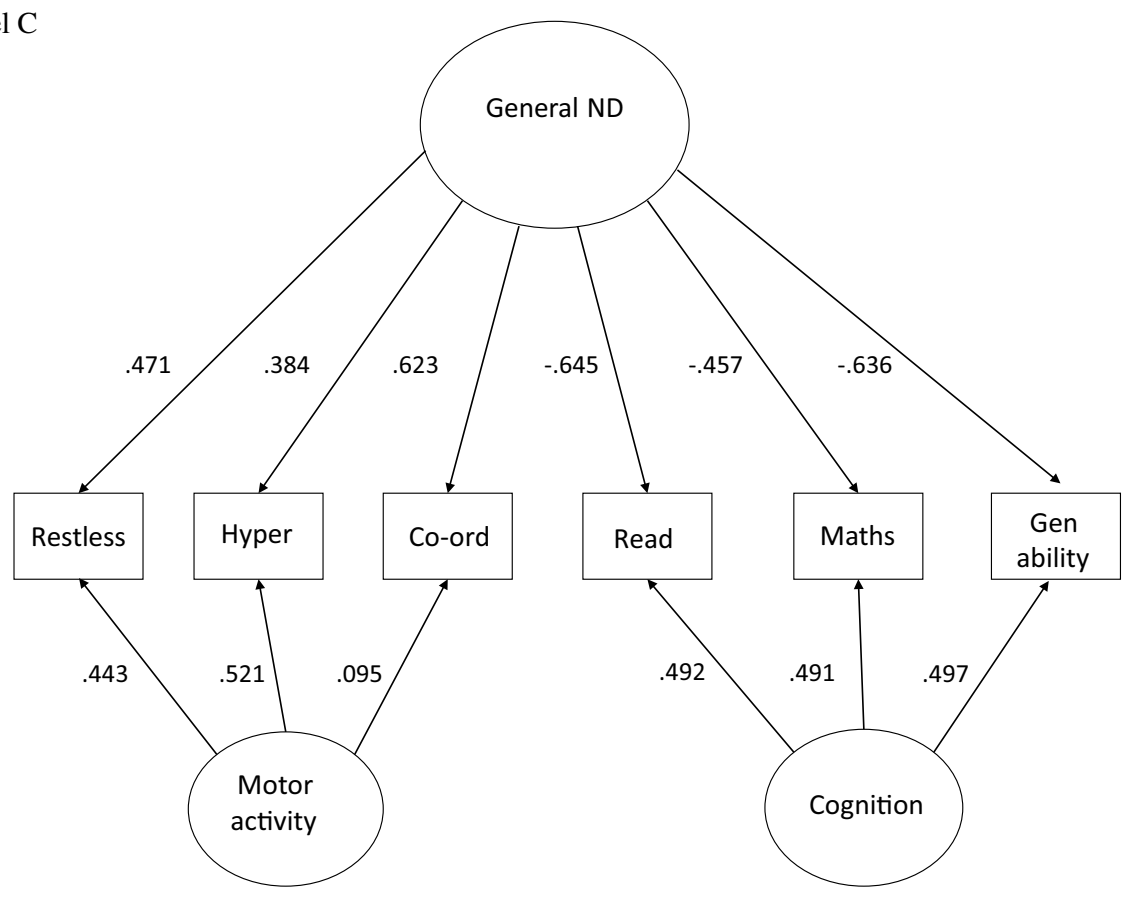


Table 1 Associations of general, motor and cognition factor scores with adult mood problems (23, 33, 42, and 50 years)

\begin{tabular}{lllll}
\hline & Mood problems 23 years & Mood problems 33 years & Mood problems 42 years & Mood problems 50 years \\
\hline Male & $N=4984$ & $N=4526$ & $N=4505$ & $N=3871$ \\
General factor & $\beta=0.166[0.136,0.196]^{* * *}$ & $\beta=0.160[0.125,0.194]^{* * *}$ & $\beta=0.144[0.107,0.181]^{* * *}$ & $\beta=0.160[0.120,0.199]^{* * *}$ \\
Motor & $\beta=-0.012[-0.039,0.016]$ & $\beta=-0.011[-0.042,0.020]$ & $\beta=0.016[-0.017,0.050]$ & $\beta=0.007[-0.029,0.042]$ \\
Cognition & $\beta=-0.040[-0.070,-0.009]^{* *}$ & $\beta=-0.012[-0.047,0.022]$ & $\beta=0.002[-0.035,0.039]$ & $\beta=-0.006[-0.044,0.033]$ \\
Female & $N=4729$ & $N=4302$ & $N=4283$ & $N=3670$ \\
General factor & $\beta=0.250[0.197,0.304]^{* * *}$ & $\beta=0.255[0.199,0.311]^{* * *}$ & $\beta=0.138[0.084,0.193]^{* * *}$ & $\beta=0.156[0.097,0.215]^{* * *}$ \\
Motor & $\beta=0.082[0.037,0.127]^{* * *}$ & $\beta=0.044[-0.002,0.090]$ & $\beta=0.050[0.005,0.095]^{*}$ & $\beta=0.029[-0.020,0.078]$ \\
Cognition & $\beta=-0.099[-0.148,-0.051]^{* * *}$ & $\beta=-0.045[-0.096,0.006]$ & $\beta=-0.061[-0.111,-0.012]^{*}$ & $\beta=-0.036[-0.090,0.019]$ \\
\hline
\end{tabular}

Sweep-specific inverse probability weights included to account for measured childhood predictors of adult non-response

${ }^{*} p<0.05 ; * * p<0.01 ; * * * p<0.001$

problems at all ages for males and females. Additional tests of the interaction by sex indicated stronger associations with mood problems for women than men at ages 23 years $(\beta=0.09, p=0.005)$ and 33 years $(\beta=0.10, p=0.003)$, but not at 42 or 50 years years $(\beta \mathrm{s}<0.01, p \mathrm{~s}>0.8)$. The cognition and motor factors in women and cognition in men showed independent associations with mood problems at age 23 years.

Findings of sensitivity analyses adjusting for child conduct and emotional problems were broadly consistent (Table 2). In these analyses, associations between the general factor and mood problems were robust across adulthood, cognition was additionally associated with mood problems at age 23 years, but there were no longer any independent associations with motor problems.

\section{Discussion}

This study set out to examine associations between neurodevelopmental traits and adult mood problems in a population-based cohort. We started by assessing whether frequently co-occurring neurodevelopmental traits were underpinned by a common neurodevelopmental factor in childhood and, moreover, whether this general factor was associated with mood problems throughout adulthood. The study also investigated whether motor-related traits and cognition showed unique associations with adult mood outcomes.

As hypothesised, the six observed measures (hyperactivity, restlessness, co-ordination, reading, arithmetic and general cognitive ability) did load on a common general neurodevelopmental factor, in keeping with previous

Table 2 Associations of general, motor and cognition factor scores with adult mood problems (23, 33, 42, and 50 years). Analyses adjusted for child emotional and conduct problems

\begin{tabular}{|c|c|c|c|c|}
\hline & Mood problems 23 years & Mood problems 33 years & Mood problems 42 years & Mood problems 50 years \\
\hline Males & $N=4784$ & $N=4340$ & $N=4335$ & $N=3728$ \\
\hline General factor & $\beta=0.159[0.129,0.190]^{* * *}$ & $\beta=0.161[0.126,0.197]^{* * *}$ & $\beta=0.147[0.109,0.185]^{* * *}$ & $\beta=0.162[0.122,0.203] * * *$ \\
\hline Motor & $\beta=-0.008[-0.036,0.020]$ & $\beta=-0.020[-0.052,0.013]$ & $\beta=-0.008[-0.043,0.027]$ & $\beta=-0.008[-0.045,0.029]$ \\
\hline Cognition & $\beta=-0.033[-0.064,-0.003]^{*}$ & $\beta=-0.006[-0.041,0.029]$ & $\beta=0.019[-0.019,0.056]$ & $\beta=0.005[-0.034,0.044]$ \\
\hline \multicolumn{5}{|l|}{ Covariates } \\
\hline Emotional probs, 7 & $\beta=0.009[-0.014,0.031]$ & $\beta=0.006[-0.020,0.032]$ & $\beta=0.010[-0.019,0.038]$ & $\beta=0.017[-0.012,0.046]^{* * *}$ \\
\hline Conduct probs, 7 & $\beta=0.063[0.039,0.087]^{* * *}$ & $\beta=0.035[0.007,0.063]^{*}$ & $\beta=0.061[0.031,0.090]^{* *}$ & $\beta=0.039[0.008,0.070]^{*}$ \\
\hline Females & $N=4545$ & $N=4127$ & $N=4116$ & $N=3524$ \\
\hline General factor & $\beta=0.245[0.191,0.299]^{* * *}$ & $\beta=0.245[0.188,0.302]^{* * *}$ & $\beta=0.134[0.079,0.190]^{* * *}$ & $\beta=0.133[0.073,0.193]^{* * *}$ \\
\hline Motor & $\beta=0.042[-0.004,0.088]$ & $\beta=0.016[-0.032,0.064]$ & $\beta=0.030[-0.017,0.077]$ & $\beta=0.014[-0.037,0.065]$ \\
\hline Cognition & $\beta=-0.077[-0.126,-0.028]^{* *}$ & $* \beta=-0.033[-0.084,0.019]$ & $\beta=-0.052[-0.102,-0.002]^{*}$ & $\beta=-0.043[-0.098,0.013]$ \\
\hline \multicolumn{5}{|l|}{ Covariates } \\
\hline Emotional probs, 7 & $\beta=0.062[0.031,0.093]^{* * *}$ & $\beta=0.035[0.002,0.069]^{*}$ & $\beta=0.050[0.017,0.082]^{* *}$ & $\beta=0.073[0.036,0.109]^{* * *}$ \\
\hline Conduct probs, 7 & $\beta=0.125[0.090,0.159]^{* * *}$ & $\beta=0.075[0.038,0.111]^{* * *}$ & $\beta=0.024[-0.012,0.059]$ & $\beta=0.035[-0.005,0.074]$ \\
\hline
\end{tabular}

Sweep-specific inverse probability weights included to account for childhood predictors of adult non-response

${ }^{*} p<0.05 ; * * p<0.01 ; * * * p<0.001$ 
research $[15,30]$. The latent structure underpinning neurodevelopmental symptom manifestation also involved more specific components, in this study motor and cognition, again consistent with prior findings [15].

The general neurodevelopmental factor was associated with mood problems throughout adult life, and this association showed little attenuation up to age 50 . There are several potential explanations for this finding. One possibility is that the general factor indexes multi-morbidity and more pronounced neurodevelopmental impairment across a range of domains, and that it is this that accounts for associations with future mood problems. Second, the general factor may index relatively stable etiological factors. For example, evidence from a recent large whole-population twin study indicates that shared genetic factors contribute strongly to phenotypic covariation across neurodevelopmental domains [15]. A third possibility is that findings reflect a more general liability for psychopathology [12]. However, there was little attenuation in associations after adjusting for co-occurring childhood emotional and conduct problems.

The study also provided some evidence for a unique contribution of specific cognition and motor factors to risk for adult mood problems over above the general factor, but the associations attenuated after early adulthood, and in the case of motor problems when adjusting for childhood emotional and conduct problems. Why associations diminished with time is unclear, but it may be that isolated motor and cognitive deficits improve with maturity, or that such problems have a greater bearing on functioning during the transition to adulthood. Previous evidence has highlighted the strong persistence of learning problems across childhood, adolescence and adult life [31,32] and there is a considerable body of research highlighting links between lower childhood educational attainment and intellectual ability and adult mood problems [33, 34]. What this study suggests is that higher rates of mood problems across the life course appear to reflect a general liability shared across ND problems, rather than liability associated with cognitive ability specifically.

The study also indicates that the association between neurodevelopmental problems and mood problems may be more pronounced in women than men, but only in early adulthood. Further research is needed to confirm this finding, but it is consistent with other reports that neurodevelopmental difficulties, at least when clinically referred, may index greater risk for depression liability in females compared to males [35].

The study extends our understanding of factors associated with risk of adult mood problems in the general population by capitalising on one of the longest prospective follow-ups of an unselected population cohort. The study included multi-informant reports of neurodevelopmental measures in childhood, and repeated assessment of mood problems in adulthood. There are also limitations that reflect the historical nature of the study. First, measures of neurodevelopmental problems were more limited. In particular, ASD-related problems, including social communication deficits and restricted repetitive behaviours, were not assessed. It is known that ASD-related traits index general ND liability [30], that ASD and other ND disorders share genetic liability [36], and that ASD traits are associated with concurrent and future mood problems $[5,8]$. A second limitation relates to those with more severe neurodevelopmental impairments. These children are likely under-represented in the cohort, and assessments of reading, maths and ability were based on group-based classroom tests. These tests are able to distinguish children with mild-to-moderate learning difficulties but those with severe learning and intellectual impairments were often unable to complete these tests [33]. Third, attrition is a challenge for all longitudinal cohorts. Participants retained in NCDS under-represented men, those with childhood neurodevelopmental problems, and those from more deprived family backgrounds [27]. Studies have also suggested that cohort members at higher neurodevelopmental genetic risk are less likely to participate in research such as this [37]. Analyses of links with adult mood did incorporate inverse probability weights to address selective drop-out related to measured childhood predictors [27]. Findings may nevertheless represent conservative estimates if those with more severe impairments in childhood and at highest risk for depression in adulthood are under-represented. Fourth, it is important to bear in mind that the findings relate to children growing up with neurodevelopmental problems in the 1960s and 1970s. Such problems are better recognised today, and there may be more support services and treatment available for children than in the past. However, the majority of children with neurodevelopmental problems still do not receive adequate help, and where help is available it is usually to deal with core neurodevelopmental deficits rather than associated mood-related problems. Finally, genetically sensitive designs together with measures of hypothesised psychosocial mediators are needed to test risk mechanisms that account for associations between neurodevelopmental problems and adult mood.

In conclusion, long-term associations between specific childhood neurodevelopmental symptoms and adult mood problems through to mid-life largely reflected associations with a more general neurodevelopmental factor. Associations with this general factor were not explained by cooccurring emotional and conduct problems in childhood, and they remained stable across adulthood. In contrast, this study found less evidence for the contribution of measured specific motor and cognitive components to long-term risk of adult mood problems. These findings highlight the importance of 
considering the joint contribution of neurodevelopmental symptoms as risk factors for long-term mood problems.

Acknowledgements We are grateful towards the National Child Development Study participants and to the UK data archive for providing us with the anonymised data. LR is supported by the Wellcome Trust (Grant ref: 204895/Z/16/Z).

\section{Compliance with ethical standards}

Conflict of interest The authors declare that they have no conflict of interest.

Ethical statement All data sets used in the present study were accessed via the UK data archive, and are covered by the study's original research ethical approvals [18].

Open Access This article is distributed under the terms of the Creative Commons Attribution 4.0 International License (http://creativeco mmons.org/licenses/by/4.0/), which permits unrestricted use, distribution, and reproduction in any medium, provided you give appropriate credit to the original author(s) and the source, provide a link to the Creative Commons license, and indicate if changes were made.

\section{References}

1. Thapar A, Cooper M, Rutter M (2017) Neurodevelopmental disorders. Lancet Psychiatry 4(4):339-346

2. Thapar A, Rutter M (2015) Neurodevelopmental disorders. In: Thapar A, Pine D, Leckman JF, Scott S, Snowling MJ, Taylor E (eds) Rutter's child and adolescent psychiatry, 6th edn. Wiley Blackwell, Chichester

3. Klein RG, Mannuzza S, Olazagasti MAR, Roizen E, Hutchison JA, Lashua EC, Castellanos FX (2012) Clinical and functional outcome of childhood attention-deficit/hyperactivity disorder 33 years later. Arch Gen Psychiatry 69(12):1295-1303

4. Clegg J, Hollis C, Mawhood L, Rutter M (2005) Developmental language disorders - a follow up in later adult life. Cognitive, language and psychosocial outcomes. J Child Psychol Psychiatry 46(2):128-149

5. Moss P, Howlin P, Savage S, Bolton P, Rutter M (2015) Self and informant reports of mental health difficulties among adults with autism findings from a long-term follow-up study. Autism 19(7):832-841

6. Biederman J, Petty CR, Monuteaux MC, Fried R, Byrne D, Mirto T, Spencer T, Wilens TE, Faraone SV (2010) Adult psychiatric outcomes of girls with attention deficit hyperactivity disorder: 11-year follow-up in a longitudinal case-control study. Am J Psychiatry 167(4):409-417

7. Meinzer MC, Pettit JR, Waxmonsky JG, Gnagy E, Molina BSG, Pelham WE (2016) Does childhood attention-deficit/hyperactivity disorder (ADHD) predict levels of depressive symptoms during emerging adulthood? J Abnorm Child Psychol 44(4):787-797

8. Hutton J, Goode S, Murphy M, Le Couteur A, Rutter M (2008) New-onset psychiatric disorders in individuals with autism. Autism 12(4):373-390

9. Vos T, Flaxman AD, Naghavi M, Lozano R, Michaud C, Ezzati $\mathrm{M}$ et al (2012) Years lived with disability (YLDs) for 1160 sequelae of 289 diseases and injuries 1990-2010: a systematic analysis for the Global Burden of Disease Study 2010. The Lancet 380(9859):2163-2196
10. Stice E, Shaw H, Bohon C, Marti CN, Rohde P (2009) A metaanalytic review of depression prevention programs for children and adolescents: factors that predict magnitude of intervention effects. J Consult Clin Psychol 77(3):486

11. Caspi A, Houts RM, Belsky DW, Goldman-Mellor SJ, Harrington H, Israel S, Meier MH, Ramrakha S, Shalev I, Poulton R, Moffitt TE (2014) The $p$ factor: one general psychopathology factor in the structure of psychiatric disorders? Clin Psychol Sci 2(2):119-137

12. Caspi A, Moffitt TE (2018) All for one and one for all: mental disorders in one dimension. Am J Psychiatry 175(9):831-844

13. Brikell I, Larsson H, Pettersson E, Chen Q, Kuja-Halkola R et al (2018) The contribution of common genetic risk variants for ADHD to a general factor of child psychopathology. Mol Psychiatry. https://doi.org/10.1038/s41380-018-0109-2

14. Martin J, Hamshere ML, Stergiakouli E, O'donovan MC, Thapar A (2015) Neurocognitive abilities in the general population and composite genetic risk scores for attention-deficit hyperactivity disorder. J Child Psychol Psychiatry 56(6):648-656

15. Pettersson E, Anckarsäter H, Gillberg C, Lichtenstein P (2013) Different neurodevelopmental symptoms have a common genetic etiology. J Child Psychol Psychiatry 54(12):1356-1365

16. Faraone S, Biederman MD, Mick E (2006) The age-dependent decline of attention deficit hyperactivity disorder: a meta-analysis of follow-up studies. Psychol Med 36(2):159-165

17. Power C, Elliott J (2005) Cohort profile: 1958 British birth cohort (National Child Development Study). Int J Epidemiol 35(1):34-41

18. Shepherd P (2012) 1958 National child development study. Ethical review and consent. London: Institute of Education, London

19. Stott DH, Sykes EG, Marston NC (1974) The social adjustment of children: manual of the British social-adjustment guides. Hodder \& Stoughton, London

20. Crow TJ, Done DJ, Sacker A (1995) Childhood precursors of psychosis as clues to its evolutionary origins. Eur Arch Psychiatry Clin Neurosci 245(2):61-69

21. Rutter M, Tizard J, Whitmore K (1970) Education, health and behaviour. Longman Publishing Group, Harlow

22. Sigurdsson E, Van Os J, Fombonne E (2002) Are impaired childhood motor skills a risk factor for adolescent anxiety? Results from the 1958 UK birth cohort and the National Child Development Study. Am J Psychiatry 159(6):1044-1046

23. Southgate V (1962) Southgate reading tests: manual of instructions. University of London Press, London

24. Pringle MLK, Butler NR, Davie R (1966) 11,000 seven year olds. Longman, London

25. Pigeon DA (1964) Tests used in the 1954 and 1957 surveys. The home and the school. MacGibbon \& Kee, London, pp 129-132

26. Rodgers B, Pickles A, Power C, Collishaw S, Maughan B (1999) Validity of the malaise inventory in general population samples. Soc Psychiatry Psychiatr Epidemiol 34(6):333-341

27. Stuart-Smith J, Thapar A, Maughan B, Thapar A, Collishaw S (2017) Childhood hyperactivity and mood problems at midlife: evidence from a prospective birth cohort. Soc Psychiatry Psychiatr Epidemiol 52(1):87-94

28. Takizawa R, Maughan B, Arsenault L (2014) Adult health outcomes of childhood bullying victimization: evidence from a fivedecade longitudinal birth cohort. Am J Psychiatry 171:777-784

29. Hu L, Bentler PM (1999) Cutoff criteria for fit indexes in covariance structure analysis: conventional criteria versus new alternatives. Struct Equ Model Multidiscipl J 6:1-55

30. Martin J, Taylor MJ, Lichtenstein P (2018) Assessing the evidence for shared genetic risks across psychiatric disorders and traits. Psychol Med 48(11):1759-1774

31. Maughan B, Messer J, Collishaw S, Pickles A, Snowling M, Yule W, Rutter M (2009) Persistence of literacy problems: spelling in adolescents and at mid-life. J Child Psychol Psychiatry 50(8):893-901 
32. Stein DS, Blum NJ, Barbaresi WJ (2011) Developmental and behavioural disorders through the life span. Pediatrics 128:364-373

33. Maughan B, Collishaw S, Pickles A (1999) Mild mental retardation: psychosocial functioning in adulthood. Psychol Med 29(2):351-366

34. Cederlöf M, Maughan B, Larsson H, D'Onofrio BM, Plomin R (2017) Reading problems and major mental disorders-co-occurrences and familial overlaps in a Swedish nationwide cohort. J Psychiatr Res 91:124-129

35. Martin J, Taylor MJ, Rydell M, Riglin L, Eyre O, Lu Y, Lundströ̀m S, Larsson H, Thapar A, Lichtenstein P (2018) Sex-specific manifestation of genetic risk for attention deficit hyperactivity disorder in the general population. J Child Psychol Psychiatry 59:908-916
36. Ghirardi L, Brikell I, Kuja-Halkola R, Freitag CM, Franke B, Asherson P, Larsson H (2018) The familial co-aggregation of ASD and ADHD: a register-based cohort study. Mol Psychiatry 23(2):257

37. Martin J, Tilling K, Hubbard L, Stergiakouli E, O'Donovan M, Davey Smith G, Thapar A, Zammit S (2015) Genetic risk for schizophrenia associated with non-participation over time in a population-based cohort study. Behav Genet 2015:45 